\title{
Quantitative molecular virology in patient management
}

\author{
Wolfgang Preiser, Bianca Elzinger, Nicola S Brink
}

The detection of viral genome (that is, viral nucleic acid, either DNA or RNA) has gained enormous importance over the last decade in the diagnosis and management of viral infections and diseases (table 1). Nucleic acid testing (NAT) has proven its superiority over more conventional laboratory techniques in several areas, for example:

- for viruses that cannot be cultured in conventional cell culture systems or those that are hazardous to isolate;

- for clinical sample material of small volume or containing only small amounts of virus;

- where antibody seroconversion is delayed after an acute infection;

- in the immunocompromised patient who may have a suboptimal antibody response;

- for the diagnosis of congenital or perinatally acquired viral infections.

As well as qualitative analysis, the characterisation of viruses by analysis of the amplified region of the genome allows the detection and identification of viral types or subtypes and mutants. This is now used to monitor patients on highly active antiretroviral therapy (HAART), based on the multitude of resistance associated mutations that have been characterised in HIV infected patients receiving antiretroviral treatment. ${ }^{1}$ This allows a more rational therapeutic approach. Sequence analysis has also proved to be a valuable epidemiological tool-for example, to investigate possible links between cases of infection, as in outbreaks of hepatitis $\mathrm{B}^{2}$ and hepatitis C infection. ${ }^{3}$

In addition to qualitative analysis and genome sequencing, methods have been developed for the quantification of viral genomes. While initially largely applied in the research field, quantitative NAT has recently been introduced into routine diagnostic virology. In this review, we will briefly introduce the different methods available for viral genome quantification, define their place in clinical virology,

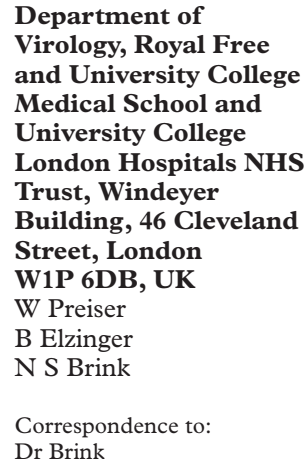

Table 1 Detection of viral genome (qualitative): clinical applications

- Detection of viruses that cannot easily be cultured: for example, hepatitis B, human papillomavirus, HIV, hepatitis $C$

- Where maximum sensitivity for viral detection is required: for example, ocular, amniotic, cerebrospinal fluid for presence of herpes simplex, varicella-zoster, ${ }^{\mathcal{C}}$ virus, cytomegalovirus, and rubella virus

- Where antibody testing is inappropriate: for example, in a primary infection (before antibody seroconversion; where passively acquired antibody may complicate the diagnosis in babies born to HCV or HIV infected mothers or in immunosuppressed patients

- To exclude infectivity: for example of blood and blood products and speculate on the possible future role of quantitative genomic testing for viral infections.

\section{Detection and quantification of viral genome: general considerations}

In principle, molecular biological assays that test for nucleic acid consist of three components:

- "front end": sample preparation, often encompassing extraction and purification of nucleic acids from the clinical material;

- "middle part": hybridisation to the target nucleic acid sequence; with genome amplification techniques this is where multiplication of the target sequence occurs;

- "back end": detection and possible quantification of target nucleic acid sequence; signal enhancement techniques use this step for signal amplification.

One of the most important objectives of the front end is the removal of inhibitors of the subsequent steps; this is particularly important with amplification techniques employing enzymatic reactions which may be inhibited by substances such as haemoglobin, heparin, and bilirubin. Another important feature is the efficiency of nucleic acid recovery. Some protocols "enrich" nucleic acid before extraction - for example, by centrifugation of the specimen to concentrate its virus content. The front end must be chosen according to the type of clinical specimen (whole blood, plasma, urine, and so on), its quantity and quality, and must also take into account the type of viral nucleic acid to be detected (DNA or RNA) and its location (intracellular or extracellular). In the context of introducing molecular techniques into a routine diagnostic service, ease of handling, speed, number of samples to be extracted per run, reproducibility, and cost are obviously important considerations. The traditional method of phenol-chloroform extraction has in some instances been replaced by faster and more easily handled methods, for example those based on nucleic acid binding to silica.

Following sample preparation and extraction of the nucleic acid, different techniques are available for detection and quantification of the target DNA or RNA. Quantification techniques include:

- target amplification techniques, for example polymerase chain reaction based assays and nucleic acid sequence based amplification (NASBA); and

- signal amplification techniques, for example branched DNA assays and hybrid capture assays. 


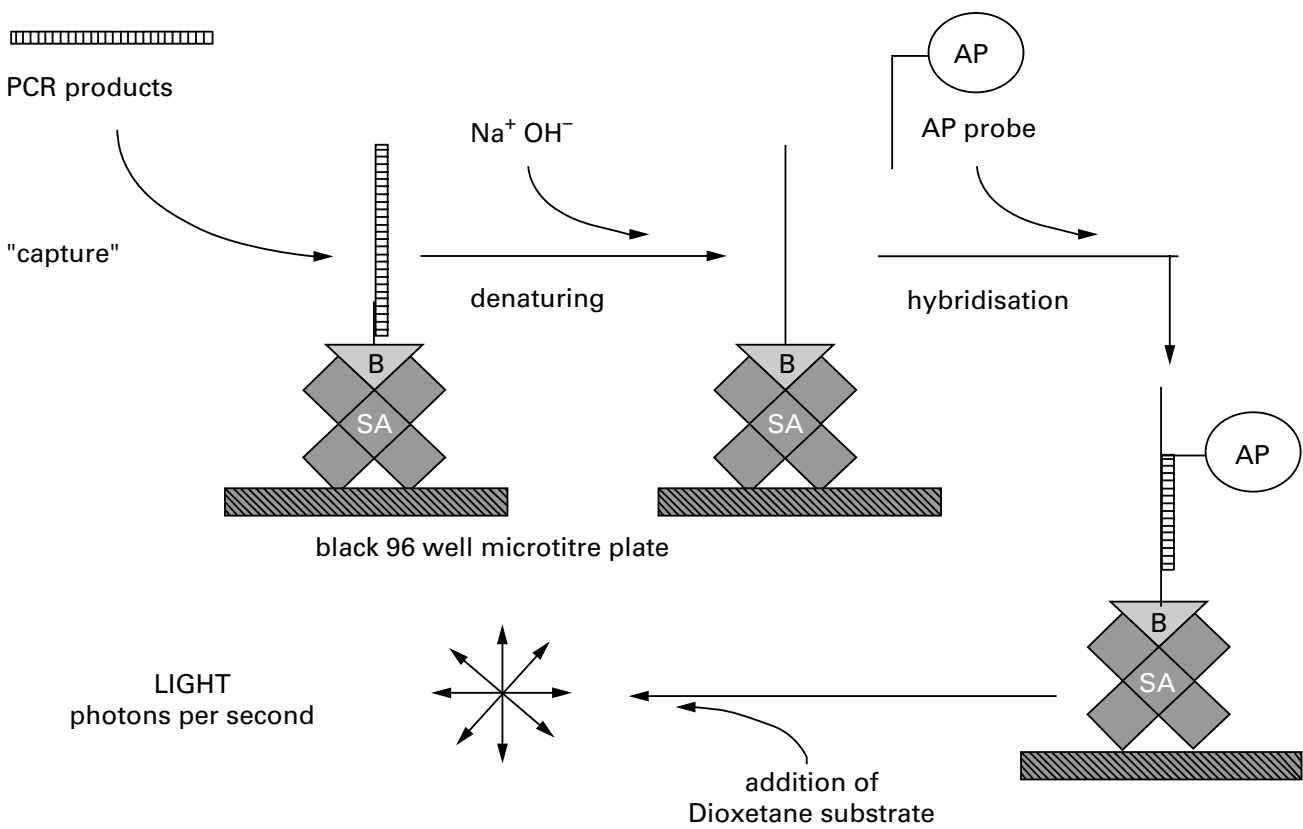

Figure 1 Principle of the enzyme linked oligonucleotide assay (ELONA) for quantification of PCR products. AP, alkaline phosphatase; B, biotin; PCR, polymerase chain reaction; $S A$, streptavidin.

\section{Quantification of viral genome: specific methods}

TARGET AMPLIFICATION: POLYMERASE CHAIN REACTION

Methods that amplify the target sequence rely on enzymatic actions to increase specifically the number of target sequence copies in the sample. This requires a careful laboratory set up and stringent practices to prevent contamination by carryover of the end product (containing possibly several million times the original amount of target sequence) into other samples or reagents, causing false positive results.

The most widely used technique is the polymerase chain reaction (PCR). Briefly, a DNA target sequence-in the case of RNA viruses (or viral messenger RNA) the RNA is first reverse transcribed into complementary DNA (reverse transcription or RT-PCR) - is amplified exponentially by a series of duplicating reactions. Methods of genome quantification using PCR based assays include:

- limiting end point dilutions of the PCR products;

- amplification with external standards;

- amplification with internal standards;

- amplification using a combination of internal and external standards;

- competitive amplification methods. ${ }^{4}$

Quantification of viral genome by "limit dilution" measures the amount of target sequence present in a sample by subjecting it at different dilutions to PCR and then detecting the presence of amplicons by electrophoresis on an ethidium bromide stained agarose gel. This method of viral genome quantification is labour intensive and slow. Quantification may also be achieved by means of standards (calibrators) of known concentration, allowing the construction of a calibration curve against which the signal obtained from the samples is plotted. The calibrators are normally run on the same test run with the samples to be analysed and may be "external" or "internal" standards, depending on whether they undergo amplification in separate reaction tubes or whether they are added to each sample in the same test tubes. A combination of internal and external calibrators may also be used. Competitive PCR is based on the comparison of the amounts of unknown target sequence produced in relation to a known quantity of a competitive template. ${ }^{4}$ Competitive PCR based quantification assays have been developed for a variety of viruses including cytomegalovirus, Kaposi's sarcoma associated herpesvirus (HHV-8), ${ }^{5}$ and hepatitis C virus (HCV). ${ }^{6}$

Methods of signal detection play an integral role in the accuracy of PCR based quantification assays. The optimal time for signal detection is to measure the amount of amplicon generated before the "plateau" phase of amplification is reached-that is, while the reaction is still in its exponential phase and the amplicon concentration proportional to the amount of target sequence at the start. This determines the range over which accurate quantification can be achieved ("dynamic range", mostly expressed in $\log _{10}$ steps). Detection is often achieved in a microtitre plate format by means of specific binding of enzyme labelled reagents to the amplified sequence; these can be complementary nucleic acid sequences (probes), anti-nucleic acid antibodies or a biotin-streptavidin system. Finally, an enzymatic reaction leads to a quantifiable colorimetric or chemiluminescent signal (analogous to the enzyme linked immunosorbent assay (ELISA) for the detection of antigen-antibody reactions). ${ }^{7}$ This is illustrated in fig 1 .

Recent additions to quantitative PCR methods are the LightCycler ${ }^{\mathrm{TM}}$ technology (Idaho Technologies Inc, Roche Molecular Biochemicals) and TaqMan ${ }^{\circledR}$ chemistry (Perkin Elmer Biosystems), combining rapid thermal cycling 
for PCR and real time detection of the reaction kinetics by means of fluorimetry.

With LightCycler, there are several possibilities for kinetic rather than end point DNA quantification: a DNA binding dye (for example, SYBR Green I) binding to the newly synthesised double stranded DNA and thereby generating a fluorescent signal proportional to its concentration, with DNA melting curve analysis allowing confirmation of the identity of the amplicon; or two hybridisation probes binding to adjacent sequences within the amplicon leaving one base in between, thus allowing fluorescence resonance energy transfer (FRET) between the donor fluor on the 3' end of one probe and the acceptor fluor on the 5 ' end of the other one.

With TaqMan, a hybridisation probe binds to the amplicon during PCR but is degraded by the nuclease activity of the Taq polymerase extending the primer; this increases the distance between the quencher and the reporter dyes with which the probe is labelled, so that the emission energy of the reporter dye is no longer transferred to the quencher and the fluorescent emission intensity increases.

Quantitative PCR assays are commercially available for cell-free HIV (HIV RNA in plasma), hepatitis C (HCV RNA in serum or plasma) and B viruses (HBV DNA in serum or plasma), as well as for cytomegalovirus (CMV DNA in leucocytes or plasma) (Amplicor HIV-1, HCV, HBV, CMV Monitor ${ }^{\mathrm{TM}}$, Roche Diagnostics). The use of the nucleotide dUTP together with the enzyme UNG (AmpErase ${ }^{\circledR}$ ) helps to prevent contamination by amplicons. The HIV-1 and HCV monitor assays initially had a 4 log dynamic range able to detect down to 400 copies/ml of HIV-1 RNA and 2000 copies/ml of HCV RNA. However, recent improvements in the assays have allowed for the detection of as low as 20 copies $/ \mathrm{ml}$ of HIV-1 RNA and 600 copies/ml of HCV RNA . Future developments include the automation of quantification assays. Roche Diagnostics have developed the Cobas AmplicorTM benchtop analyser which incorporates thermal cycling, pipetting, incubation, washing, and reading.

\section{TARGET AMPLIFICATION: NUCLEIC ACID}

SEQUENCE BASED AMPLIFICATION

Nucleic acid sequence based amplification (NASBA) is an isothermal RNA amplification reaction which makes use of three different enzymes active at $41^{\circ} \mathrm{C}$ : AMV-RT (avian myeloblastosis virus reverse transcriptase), RNAse H, and T7 RNA polymerase. Primer 1, containing a T7 RNA polymerase promoter sequence, anneals to the target sequence, single stranded RNA, and is used by the reverse transcriptase to form an RNA:DNA hybrid. The RNA strand is then degraded by the action of RNAse $\mathrm{H}$ and a second DNA strand synthesised by extension of primer 2 by the reverse transcriptase. If the target sequence is DNA, this is heat denatured to allow attachment of primer 1 and synthesis of a double stranded DNA carrying the $\mathrm{T} 7$ promoter sequence.
Because the resulting double stranded DNA carries the additional $\mathrm{T} 7$ promoter sequence its sense strand can serve as a template for $\mathrm{T} 7$ RNA polymerase for synthesis of an RNA strand. The reaction then enters the amplification phase during which, in a cyclic manner, the newly formed RNA acts as a template for the reverse transcriptase to synthesise DNA. It is then digested again by the RNAse $\mathrm{H}$ and replaced by a complementary DNA strand which allows the T7 RNA polymerase to produce more RNA molecules entering the amplification cycle anew. Three internal calibrators that are coextracted and coamplified $(\mathrm{Q} a, \mathrm{Qb}$, and $\mathrm{Qc})$ allow for quantification. The quantity of amplified target genome and calibrators is measured using electrochemiluminescence, and the signal produced by the target genome is compared with the signals from the three calibrators. ${ }^{4}$ NASBA is marketed by Organon Teknika as part of the NucliSens system for the quantitative detection of HIV RNA (NucliSens HIV-1 QT). The assay has a dynamic range of 4 logs and could initially detect down to 400 HIV-1 RNA copies $/ \mathrm{ml}$. More recently a more sensitive assay has been developed which can detect down to 40 HIV-1 RNA copies/ml.

SIGNAL AMPLIFICATION: HYBRIDISATION TECHNIQUES

Hybridisation procedures are based on the detection of a single stranded (that is, denatured) nucleic acid sequence by means of a labelled nucleic acid probe. This probe is complementary to the genome region in question and therefore specifically binds to the target nucleic acid. This binding can then be visualised, depending on the nature of the label, by autoradiography or an enzymatic reaction, leading to a colour change.

Quantitative hybrid capture ${ }^{\mathrm{TM}}$ systems (Digene Corporation; Murex) are commercially available for human cytomegalovirus DNA in leucocytes and hepatitis B virus (HBV) DNA in serum. These assays use RNA probes which bind to the target DNA from the clinical specimen to form RNA:DNA hybrids. These are captured onto a solid phase coated with anti-RNA:DNA hybrid capture antibodies. The same antibodies labelled with the enzyme alkaline phosphatase are then used to bind to the solid phase bound RNA:DNA hybrids ("sandwich" style immunoassay). Finally, the amount of bound alkaline phosphatase is measured quantitatively by addition of a chemiluminescent substrate and counting of photons emitted. Because several alkaline phosphatase molecules are conjugated to each anti-RNA:DNA hybrid antibody and because multiple conjugated antibodies bind to each captured hybrid molecule, a 3000-fold or higher signal amplification is achieved (fig 2).

SIGNAL AMPLIFICATION TECHNIQUES: BRANCH DNA ASSAY

With branch DNA (bDNA) assays (Quantiplex $^{\circledR}$, Chiron Diagnostics) a combination of synthetic oligonucleotide probes binds to and 


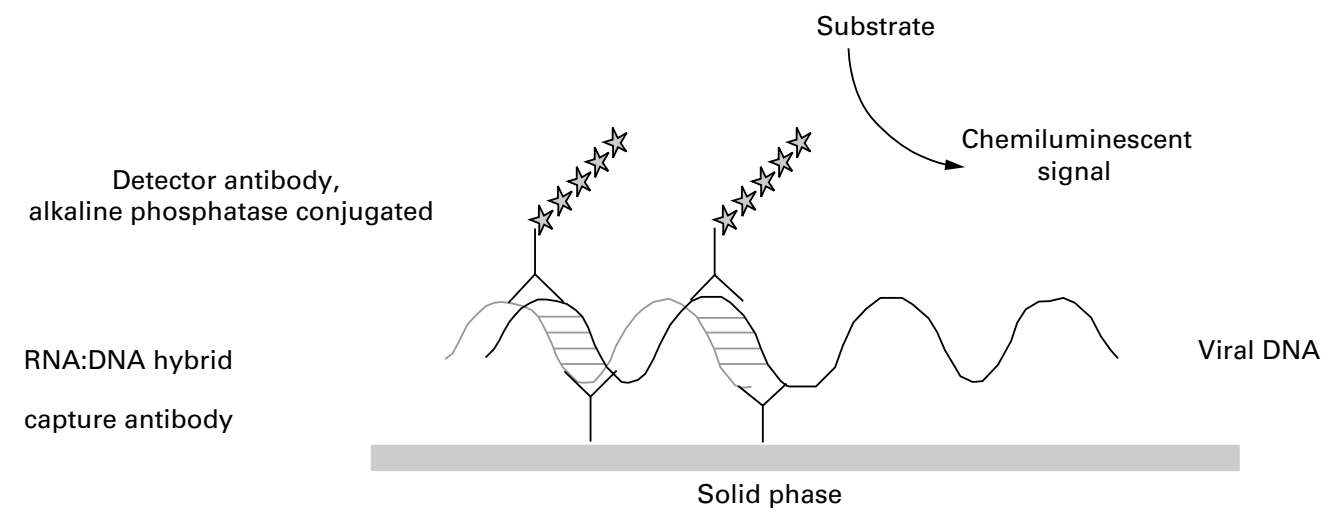

Figure 2 Signal amplification: hybrid capture assay.

measures the amount of viral RNA or DNA in the sample. Target viral nucleic acid is captured onto a solid phase by means of oligonucleotide probes. Several other species of probes then bind to the target sequence and to each other in a sequential fashion, including branched DNA (bDNA) amplifier probes. Finally, multiple copies of the alkaline phosphatase labelled probe hybridise to each amplifier probe on the immobilised complex; addition of a chemiluminescent substrate cleaved by alkaline phosphatase then leads to light emission proportional to the amount of target sequence in the sample (fig 3).

Currently available are Quantiplex ${ }^{\circledR}$ bDNA assays for the quantification of HIV-1 RNA, HCV RNA, and HBV DNA (Chiron Corporation). Initially the HIV-1 bDNA assay had a sensitivity of 500 copies $/ \mathrm{ml}$, but more recently an ultrasensitive version of the HIV assay has been produced which employs centrifugation to concentrate virions in the plasma before hybridisation and detects down to 50 copies/ $\mathrm{ml}$.
Table 2 Quantification of viral genome: clinical applications

Prognostic marker: for example, disease progression in HIV Therapeutic marker: for example, for the initiation and monitoring antiviral treatment

To assess the risk of infection: for example, the risk of perinatal transmission in babies born to HIV and HCV infected women

\section{Clinical applications of viral nucleic acid} quantification

The clinical applications of viral nucleic acid quantification are summarised in table 2 .

PROGNOSTIC MARKERS AND CLINICAL DISEASE ASSOCIATIONS

Viral genome quantification as a prognostic marker or an indicator of disease severity is now well established for certain viral infections, in particular HIV. A study in the late 1980s showed an association between the levels of plasma HIV (as measured by end point dilution cultures) and disease stage, with individuals with AIDS having higher levels of tissue culture infective doses of HIV per ml plasma. ${ }^{8}$ Although culture based, this study provided the

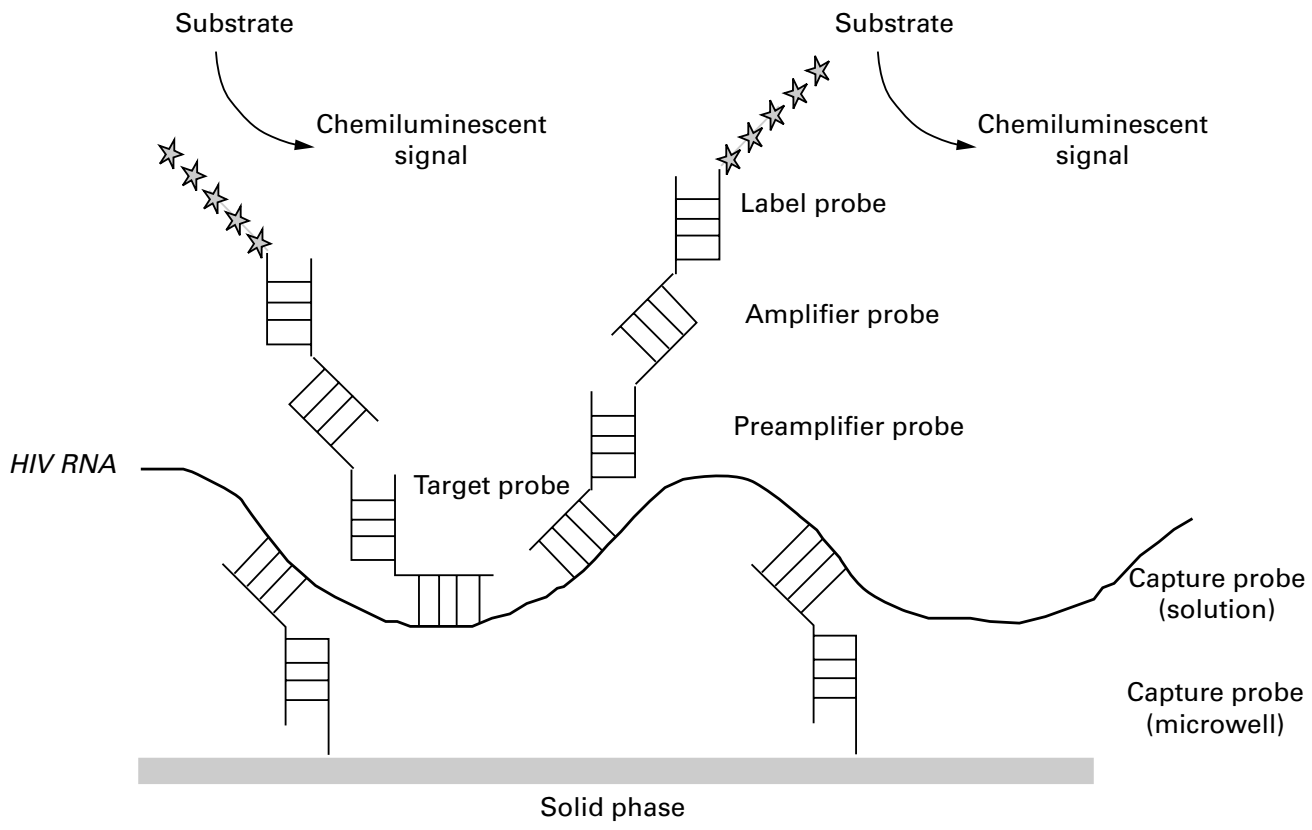

Figure 3 Signal amplification: branched DNA assay. 
first insight into the potential use of viral load as a prognostic marker. The association of plasma viral load with disease stage was confirmed in the early 1990s in a study using the direct quantification of plasma RNA, using a PCR based method. ${ }^{9}$ More recently the demonstration of low levels of HIV-1 in the majority of long term non-progressors (defined as HIV infected individuals who remain healthy and immunologically normal for more than 10 years) has provided further information on the role of HIV viral load as a prognostic marker. ${ }^{10}$ It has also been shown that the postseroconversion viral load may predict progression to AIDS. ${ }^{11}$ This study showed that a high plasma HIV-1 RNA level (100 000 copies/ml) was the most powerful predictor of AIDS. In contrast, a plasma HIV-1 RNA level of less than $10000 \mathrm{copies} / \mathrm{ml}$ was not associated with progression to AIDS. Table 3 shows the clear relation between viral load (serum HIV-1 RNA) and disease outcome in HIV infected individuals. ${ }^{12}$ It has also been found that plasma HIV-1 RNA levels are predictive of the subsequent clinical course in perinatally infected infants. ${ }^{13}$

Cytomegalovirus infection is common in immunosuppressed patients and may result in severe disease. Rapid laboratory diagnosis of infection and the ability to predict which patients are at risk of progression to clinical disease is therefore important. Qualitative cytomegalovirus DNA detection has been used in post-transplant monitoring programmes, but will inevitably involve the unnecessary treatment of certain patients if pre-emptive anti-CMV therapy is used (that is, treatment of patients with evidence of cytomegalovirus infection but not disease). A study analysing 248 blood samples from immunocompromised patients showed that the positive predictive value of qualitative PCR positivity in the blood with respect to the development of cytomegaloviral disease was $0.62 .{ }^{14}$ The initiation of preemptive anti-CMV therapy on the basis of the qualitative detection of cytomegalovirus DNA in blood therefore results in the exposure of patients to potentially toxic drugs without clinical need. Refinement of cytomegalovirus surveillance after transplantation may be achieved by the monitoring the cytomegalovirus load, and this is likely to form an integral part of post-transplantation monitoring programmes in the future. Critical here is the determination of an appropriate cut off for therapeutic intervention. Using a PCR based quantification assay, Fox and colleagues showed that high levels of cytomegalovirus correlated with clinically apparent cytomegalovirus associated disease in a cohort of renal

Table 3 Relation of plasma HIV viral load to survival times in HIV infected individuals

HIV viral load

(RNA copies/ml plasma) Median survival time

$>36270 \quad 5.1$ years

$13021-36270 \quad 7.4$ years

$4531-13020 \quad 9.5$ years

$<4530>10$ years

(from Mellors et al, $1996^{12}$ ) transplant recipients. ${ }^{15}$ A similar association was found in a group of bone marrow transplant recipients, where only cytomegalovirus viral load was a risk factor for the development of cytomegalovirus associated disease. ${ }^{16}$ In HIV infected patients it has also been shown that median cytomegalovirus viral load is significantly greater in patients who subsequently develop clinical disease. ${ }^{17}$

It has been suggested that the amount of cytomegalovirus in the urine from an infected neonate may be directly related to the prognosis of congenital cytomegalovirus infection. ${ }^{4}$ It is possible that this may be extended to the quantification of cytomegalovirus DNA in amniotic fluid as a predictor of severe fetal disease in the future.

Determination of viral load may also be useful for the diagnosis of disease associated with other herpesviruses. Post-transplant lymphoproliferative disorder (PTLD) is a potentially life threatening complication following allogeneic transplantation associated with infection with the Epstein-Barr virus (EBV). Recent studies have linked EBV viral load in plasma and peripheral blood lymphocytes to the risk of developing PTLD. ${ }^{18} 19$ This may be important in monitoring patients at risk of disease development, particularly as the current method of diagnosing PTLD is based on a combination of clinical and histopathological features, an approach which may be too slow to be clinically useful. ${ }^{4}$ Determination of EBV viral load may therefore be a useful marker to predict disease development, thereby influencing patient morbidity and possibly mortality, as management of PTLD includes the reduction of immunosuppressive drugs. Assays for the quantification of human herpesvirus 6 and 7 have also been developed and it is possible that these will help define the pathogenic role of these ubiquitous viruses. $^{2021}$

In contrast to the demonstrable value of HIV-1 and cytomegalovirus viral load as a prognostic marker in HIV infected individuals, no reliable association has been shown between serum HCV titres and the severity of liver disease. ${ }^{22}$ For hepatitis $\mathrm{B}$, however, a rough correlation between the presence of liver disease and the amount of serum HBV DNA has been demonstrated. A study showed that patients who were hepatitis B surface antigen (HBsAg) and hepatitis $\mathrm{B}$ e antigen ( $\mathrm{HBeAg}$ ) positive all had detectable HBV DNA by PCR. A majority of these patients also had HBV DNA detectable by the less sensitive dot blot hybridisation and most had significant liver disease. In contrast, patients who had detectable serum $\mathrm{HBsAg}$ but no $\mathrm{HBeAg}$, and lower mean serum transaminase levels, usually did not have detectable HBV virus DNA by dot blot hybridisation. ${ }^{23}$ It is therefore likely that the level of HBV DNA is a more accurate marker of virus replication than $\mathrm{HBeAg}$ alone and may be important in the study of the pathogenesis of $\mathrm{HBeAg}$ negative pre-core mutations. ${ }^{24}$ 
INITIATION AND MONITORING OF ANTIVIRAL TREATMENT

One of the most important clinical applications for viral nucleic acid quantification is for the initiation, monitoring, and modification of antiviral treatment. For HIV-1 the goal of antiretroviral therapy is to reduce plasma HIV-1 RNA levels to the lowest level possible for as long as possible. This is logical given the interaction between HIV-1 replication and CD4 lymphocyte destruction, which in turn influences clinical outcome. ${ }^{25}$ The first question is, at what plasma HIV-1 RNA level should treatment be initiated? It has been suggested that all patients with plasma HIV-1 RNA levels of > $30000-50000$ copies $/ \mathrm{ml}$ should be offered antiretroviral treatment regardless of the state of their clinical disease, whereas for individuals with plasma HIV-1 levels of $>5000$ but $<30000$ copies $/ \mathrm{ml}$ the decision to treat should take into account the CD4 count and clinical disease stage. ${ }^{25}$ Following initiation of antiretroviral therapy, viral load should be used to monitor the therapeutic response. At baseline this should consist of two measurements two to four weeks apart and thereafter every three to four months in a clinically stable patient. A 0.5 log or greater reduction of plasma RNA levels is considered to be the minimum response indicating an antiretroviral effect. If HIV-1 RNA levels return to (or to within 0.3-0.5 logs of) pretreatment levels, this could signify the selection of a resistant virus. ${ }^{25}$ However, other causes of a rising viral load, such as poor drug compliance or an acute opportunistic infection, should be excluded. ${ }^{26}$

Molecular quantification of $\mathrm{HBV}$ virus DNA has been used to predict a response to interferon and to monitor interferon treatment in patients infected with HBV. High pretreatment levels of virus DNA are predictive of a poor therapeutic response to interferon. ${ }^{4} \mathrm{Sev}$ eral factors influence the therapeutic response to antiviral treatment in patients infected with HCV. These include the virus genotype (patients infected with genotype 1 respond less well than patients infected with genotypes 2 or 3) and the viral load. A study by Garson et al showed that individuals who had a sustained response to antiviral treatment (defined as a complete normalisation of aminotransferase levels within three months of starting interferon, and maintained throughout the 12 month follow up period) had mean pretreatment HCV RNA levels that were approximately 10-fold lower than in individuals who did not have a sustained response. ${ }^{27}$ This was confirmed in a later study. ${ }^{28}$ In the consensus statement produced following the 1999 EASL international consensus conference on hepatitis $\mathrm{C}$, it was noted that while patients with higher levels of viraemia (more than two million copies $/ \mathrm{ml}$ ) are less likely to respond to therapy, the level of viraemia should not be used as a reason to withhold treatment. The level of viraemia does, however, influence the duration of treatment in patients infected with HCV genotype 1. Here the current data suggest that six months of treatment are sufficient for patients with less than two million copies/ml of serum HCV RNA, whereas 12 months of treatment are recommended for patients with viraemia in excess of two million copies $/ \mathrm{ml}$. Monitoring the therapeutic response is currently done by qualitative PCR, although genome quantification is likely to play an important role in the future. ${ }^{29}$ It has also been suggested that the rate of decline in viraemia during the first few weeks on interferon treatment can predict the therapeutic outcome in chronically infected patients. ${ }^{30}$

MARKERS OF INFECTIVITY

The use of viral genome quantification as a marker of infectivity is likely to play an important role in patient management in the future. Possible uses include the assessment of the risk of transmission of HCV or HIV from mother to child in the peripartum period, together with the risk of infection after occupational exposure to a bloodborne virus. Plasma or serum levels of HIV and HCV are important determinants of risk in the perinatal transmission from mother to child. For hepatitis C, mother to child transmission is more likely if the maternal serum HCV RNA concentration is greater than $10^{6}$ to $10^{7}$ genomes $/ \mathrm{ml} .^{31}$ A study comparing transmission rates from various centres showed that only two of 30 women who transmitted infection to their infants had a viral load of less than $10^{6}$ copies $/ \mathrm{ml} .{ }^{32}$ Coinfection with hepatitis $\mathrm{C}$ and HIV also increases transmission rates of HCV (from 3.7\% in patients infected with hepatitis $\mathrm{C}$ alone to $15.5 \%$ in women coinfected with HIV in one study), probably because of the increase in HCV RNA in the serum of coinfected women. ${ }^{33}$ Although a study from Italy has suggested a lower rate of transmission in babies born by caesaean section compared with vaginal delivery $(6 \% v$ $32 \%),{ }^{34}$ the optimum mode of delivery for the woman infected with $\mathrm{HCV}$ has not yet been defined. It is possible, therefore, that HCV viral load may help in the choice of delivery in the future. Although the risk of transmission of HIV-1 from mother to child is also increased with a higher viral load, a recent study could not demonstrate a threshold value of virus load that discriminated between transmitters and non-transmitters. It also showed transmission and non-transmission occurring over the entire range of each of the quantification assays used, indicating the need for caution when considering viral load as a risk factor for transmission of HIV-1 infected mothers. ${ }^{35}$ It is likely, therefore, that the prevention of perinatally acquired HIV infection will largely be based on the use of antiretroviral treatment, elective caesarean section, and the avoidance of breast feeding.

The role of viral load in assessing the risk of HIV transmission, both sexually and through occupational exposure, needs to be investigated further. A recent study examined the relation of viral load to heterosexual transmission of HIV in haemophiliac men and showed an association of high viral load with transmission of infection. This may help in the counselling of haemophiliac couples in the future. ${ }^{36}$ Although there is no prospective study determining the risk of infection with a bloodborne virus after 
occupational exposure (through needlestick accidents or mucous membrane or non-intact skin exposure) it is reasonable to assume the risk of infection is related to the quantity of virus in the source material. This in turn is influenced by the disease stage of the patient; for example it has been shown that HIV-1 viral load increases with progression to AIDS (see above).

A possible future development is the use of serum HBV levels to clear infected health care workers who are found to be "low infectivity" hepatitis B carriers (that is, their serum does not contain hepatitis B e antigen) for the performance of exposure-prone procedures. Current Department of Health guidelines in the United Kingdom state that health care workers who are $\mathrm{HBV}$ infected but are hepatitis B carriers of low infectivity can perform exposure-prone procedures as long as they have not been involved in a transmission event from health care worker to patient. ${ }^{37}$ Despite this, transmission events still occur, and seven such events have occurred in the United Kingdom in so called "low infectivity" hepatitis B carriers (Tedder R, personal communication). Addition of HBV viral load determination is likely to add an extra margin of safety, while allowing health care workers at a low risk of transmission to continue their surgical career.

\section{Conclusions}

Viral genome quantification now forms an integral part of the management of HIV infected patients, providing pretreatment prognostic information as well as monitoring a therapeutic response. The next few years are likely to see the expansion of the clinical applications of nucleic acid quantification, with its use in the monitoring of $\mathrm{HBV}$ and HCV infected patients on antiviral treatment. Blood cytomegalovirus DNA levels will also guide the use of pre-emptive anti-CMV treatment in patients following bone marrow transplantation, allowing the more rational use of potentially myelosuppressive antiviral treatment. International standardisation of molecular quantification techniques will allow comparison of results, enabling consensus policies for infectious disease management to be produced. Genome quantification may also provide an accurate assessment when evaluating the risk of infection, for example in the transmission of $\mathrm{HCV}$ from mother to child and in the HBV infected health care worker.

We thank Dr J Garson for his helpful comments and advice.

1 Hammond J, Calef C, Larder B, et al. Mutations in retroviral genes associated with drug resistance. In: Korber B, Kuiken C, Foley B, et al, eds. Human retroviruses and AIDS 1998: a compilation and analysis of nucleic acid and amino acid sequences. Los Alamos: Theoretical Biology and Biophysics Group T-10, 1998:III-36-79.

2 Hawkins AE, Zuckerman MA, Briggs M, et al. Hepatitis B nucleotide sequence analysis: linking an outbreak of acute hepatitis B to contamination of a cryopreservation tank. $\mathcal{F}$ Virol Methods 1996;60:81-8.

3 Smith DB, Lawlor E, Power J, et al. A second outbreak of hepatitis $C$ virus infection from anti-D immunoglobulin in Ireland. Vox Sanguinis 1998;76:175-80.
4 Hodinka RL. The clinical utility of viral quantification using molecular methods. Clin Diagn Virol 1998;10:25-47.

5 Lock MJ, Griffiths PD, Emery VC. Development of a quantitative polymerase chain reaction for human herpesvirus 8 . f Virol Methods 1997;64:19-26.

6 Ravaggi A, Zonaro A, Mazza C, et al. Quantitation of hepatitis C RNA by competitive amplification of RNA from denatured serum and hybridisation on microtitre plates. $\mathcal{F}$ Clin Microbiol 1995;33:265-9.

7 Whitby K, Garson JA. Optimisation and evaluation of a quantitative chemiluminescent polymerase chain reaction assay for hepatitis C RNA. F Virol Methods 1995;51:75-88.

8 Ho DD, Tarsem M, Masud A. Quantitation of human immunodeficiency virus type 1 in the blood of infected persons. N Engl f Med 1989;321:1621-5.

9 Semple MG, Kaye S, Loveday C, et al. Direct measurement of viraemia in patients infected with HIV-1 and its relationship to disease progression and zidovudine therapy. $7 \mathrm{Med}$ Virol 1991:35:38-45

10 Cao Y, Qin L, Zhang L, et al. Virologic and immunologic characterisation of long-term survivors of human immunodeficiency virus type 1 infection. $N$ Engl f Med 1995;332: 201-8

11 Mellors JW, Kingsley LA, Rinaldo CR, et al. Quantitation of HIV-1 RNA in plasma predicts outcome after seroconversion. Ann Intern Med 1995;122:573-9.

12 Mellors JW, Rinaldo CR, Gupta P, et al. Prognosis in HIV-1 infection predicted by the quantity of virus in plasma. Science 1996;272:1167-70.

13 Shearer WT, Quinn TC, LaRussa P, et al. Viral load and disease progression in infants infected with human immunodeficiency virus type 1. N Engl f Med 1997;336: 1337-42.

14 Kidd IM, Fox JC, Pillay D, et al. Provision of prognostic information in immunocompromised patients by routine application of polymerase chain reaction for cytomegaloviapplication of polymerase chain reaction $1993 ; 56: 867-71$.

15 Fox JC, Kidd IM, Griffiths PD, et al. Longitudinal analysis of cytomegalovirus load in renal transplant recipients using quantitative polymerase chain reaction: correlation with disease. F Gen Virol 1995;76:309-19.

16 Gor D, Sabin C, Prentice HG, et al. Longitudinal fluctuations in cytomegalovirus load in bone marrow transplant patients: relationship between peak virus load, donor/ recipient serostatus, acute GVHD and CMV disease. Bone Marrow Transplant 1998;21:597-605.

17 Bowen EF, Sabin CA, Wilson P, et al. Cytomegalovirus (CMV) viraemia detected by polymerase chain reaction identifies a group of HIV-positive patients at high risk of CMV disease. AIDS 1997;11:889-93.

18 Riddler SA, Breinig MC, McKnight JLC. Increased levels of circulating Epstein-Barr virus (EBV)-infected lymphocytes arculating Epstein-Barr virus (EBV)-infected lymphocytes associated with the development of posttransplant lymphoassociated with the development of posttransplant lympho-
proliferative disease in solid organ transplant recipients. proliferative disease in
Blood 1994;84:972-84

19 Yamamoto M, Kimura H, Hironaka T, et al. Detection and quantitation of virus DNA in plasma of patients with Epstein-Barr virus-associated diseases. $\mathcal{F}$ Clin Microbiol 1995;33:1765-8

20 Clark DA, Ait-Khalid M, Wheeler AC. Quantification of human herpesvirus 6 in immunocompetent and postmortem tissues from AIDS patients by OCR. 7 Gen Virol 1996;77:2271-5.

21 Kidd IM, Clark DA, Ait-Khaled M, et al. Measurement of human herpesvirus 7 load in peripheral blood and saliva of healthy subjects by quantitative polymerase chain reaction. fInfect Dis 1996;174:396-401.

22 Ahmed MM, Mutimer DJ, Martin B, et al. Hepatitis C viral load, genotype and histological severity in patients with load, genotype and histological severity in pa
bleeding disorders. Haemophilia 1999;5:49-55.

23 Baker BL, Di-Bisceglie AM, Kaneko S, et al. Determination of hepatitis B virus DNA in serum using the polymerase chain reaction: clinical significance and correlation with clinical and biochemical markers. Hepatology 1991;13:6326.

24 Berger A, Braner J, Doerr HW, et al. Quantification of viral load: clinical relevance for human immunodeficiency virus, hepatitis B virus and hepatitis $\mathrm{C}$ virus infection. Intervirology 1997;41:24-34.

25 Saag MS, Holodniy M, Kuritzkes DR, et al. HIV viral load markers in clinical practice. Nature Med 1996;2:625-9.

26 Churchill D, Weber J. Measurement of plasma HIV viral load. F Infect 1999;38:147-50.

27 Garson JA, Brillanti S, Whitby K, et al. Analysis of clinical $\mathrm{Garson} \mathrm{JA}$, Brillanti $\mathrm{S}$, Whitby $\mathrm{K}$, et al. Analysis of clinical
and virological factors associated with response to alpha and virological factors associated with response to alpha
interferon therapy in chronic hepatitis C. F Virol Methods interferon therapy

28 Garson JA, Uhnoo I, Whitby K, et al. Virological, biochemi$\mathrm{cal}$ and histological effects of human lymphoblastoid interferon in Swedish patients with chronic hepatitis C. F Viral Hepatitis 1997;4:325-31.

29 EASL International Consensus Conference on Hepatitis C. Paris 26-28 February 1999. Consensus Statement. F Hepatol 1999;30:956-61.

30 Walsh KM, Good T, Cameron S, et al. Viral kinetics can predict early response to alpha-interferon in chronic hepatitis C. Liver 1998;18:191-5.

31 Dusheiko GM, Khakoo S, Soni P, et al. A rational approach to the management of hepatitis C infection. BMF 1996;312:357-64.

32 Thomas SL, Newell ML, Peckham CS, et al. A review of hepatitis $\mathrm{C}(\mathrm{HCV})$ vertical transmission: risks of transmission to infants born with and without HCV viraemia and 
human immunodeficiency virus infection. Int 7 Epidemiol 1998;27:1208-17.

33 Tovo PA, Palomba E, Ferraris G, et al. Increased risk of maternal-infant hepatitis $\mathrm{C}$ transmission for women co-infected with human immunodeficiency virus type 1 . Italian Study Group for

34 Paccagnini S, Principi N, Massironi E, et al. Perinatal transmission and manifestation of hepatitis $\mathrm{C}$ virus infection in a high risk population. Pediatr Infect Dis f 1995;14:195-9.
35 Cao Y, Krogstad P, Korber BT, et al. Maternal HIV viral load and vertical transmission of infection: the Ariel Project for the prevention of HIV transmission from mother to infant. Nature Med 1997;3:549-52.

36 Ragni MV, Faruki H, Kingsley LA. Heterosexual HIV-1 transmission and viral load in hemophilic patients. F Acquir Immune Defic Syndr Hum Retrovirol 1998;17:42-5.

37 Health Service Guidelines. Protecting health care workers and patients from hepatitis B. London: department of Health, 1993 [HSG(93)40:19931-3]. 